\title{
Aberration and the Speed of Gravity
}

\author{
S. CARLiP \\ Department of Physics \\ University of California \\ Davis, CA 95616 \\ $U S A$
}

\begin{abstract}
The observed absence of gravitational aberration requires that "Newtonian" gravity propagate at a speed $c_{g}>2 \times 10^{10} c$. By evaluating the gravitational effect of an accelerating mass, I show that aberration in general relativity is almost exactly canceled by velocity-dependent interactions, permitting $c_{g}=c$. This cancellation is dictated by conservation laws and the quadrupole nature of gravitational radiation.
\end{abstract}

*email: carlip@dirac.ucdavis.edu 
In a recent paper in Physics Letters A [1], Van Flandern has argued that observations show that gravity propagates at a speed much greater than $c$. In the absence of direct measurements of propagation speed, Ref. [1] relies instead on directional information, in the form of observations of (the absence of) gravitational aberration. But the translation from a direction to a speed requires theoretical assumptions, and the implicit assumptions of Ref. [1] - in particular, that the interaction is purely central, with no velocity-dependent terms - do not hold for general relativity, or, for that matter, for Maxwell's electrodynamics.

In this paper, I explicitly compute the gravitational effect of an arbitrarily accelerating source, Kinnersley's "photon rocket" [2]. Although gravity propagates at the speed of light in general relativity, the expected aberration is almost exactly canceled by velocity-dependent terms in the interaction. While at first this cancellation seems to be "miraculous," it can be explained from first principles by turning Van Flandern's argument on its head: conservation of energy and angular momentum, together with the quadrupole nature of gravitational radiation, require that any causal theory have such a cancellation.

\section{Aberration in Electromagnetism}

It is certainly true, although perhaps not widely enough appreciated, that observations are incompatible with Newtonian gravity with a light-speed propagation delay added in [3, 4]. If one begins with a purely central force and puts in a finite propagation speed by hand, the forces in a two-body system no longer point toward the center of mass, and the resulting tangential accelerations make orbits drastically unstable. A simple derivation is given in problem 12.4 of Ref. [幽, where it is shown that Solar System orbits would shift substantially on a time scale on the order of a hundred years. By analyzing the motion of the Moon, Laplace concluded in 1805 that the speed of (Newtonian) gravity must be at least $7 \times 10^{6} c[5]$. Using modern astronomical observations, Van Flandern raised this limit to $2 \times 10^{10} c$ [1].

But this argument, at least in its simplest form, holds only if one postulates that the relevant force is purely central and independent of the source velocity. As Poincaré observed as early as 1905 [6,7], the effects of aberration can be drastically altered by velocity-dependent interactions. And indeed, for Maxwell's electrodynamics and Einstein's general relativity, such interactions occur [8].

As a warm-up, let us first consider electrodynamics. It is well known that if a charged source moves at a constant velocity, the electric field experienced by a test particle points toward the source's "instantaneous" position rather than its retarded position. Lorentz invariance demands that this be the case, since one may just as well think of the charge as being at rest while the test particle moves. This effect does not mean that the electric field propagates instantaneously; rather, the field of a moving charge has a velocity-dependent component that cancels the effect of propagation delay to first order [9].

It is helpful to analyze this case a bit more carefully, while establishing notation that will be useful below when we discuss general relativity. Let the source move along a timelike world line $C$ in flat Minkowski spacetime, with position $z^{\mu}(s)$ and four-velocity $\lambda^{\mu}=d z^{\mu} / d s$. The backwards light cone from any point $x^{\mu}$ will intersect $C$ at a point $z^{\mu}\left(s_{R}\right)$ (see figure 1 ), and this relation can be viewed as an implicit definition of the retarded proper time $s_{R}(x)$ : 


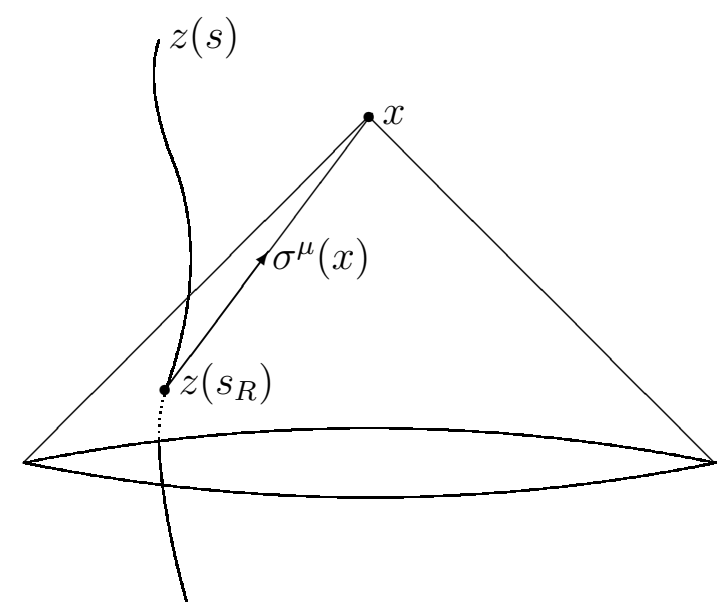

Figure 1: The geometry of retarded positions in Minkowski space

$$
\eta_{\mu \nu}\left(x^{\mu}-z^{\mu}\left(s_{R}\right)\right)\left(x^{\nu}-z^{\nu}\left(s_{R}\right)\right)=0 .
$$

Let

$$
\sigma^{\mu}=x^{\mu}-z^{\mu}\left(s_{R}\right)
$$

denote the null vector connecting $x$ and $C$. Differentiating (1.1), we obtain

$$
\partial_{\mu} s_{R}(x)=\frac{\sigma_{\mu}}{r}
$$

where

$$
r(x)=\lambda^{\mu}\left(s_{R}\right) \sigma_{\mu}
$$

is an invariant retarded distance from $x$ to $C$. In terms of a $(3+1)$-dimensional decomposition of spacetime, we have (in units $c=1$ )

$$
\begin{array}{cc}
\sigma^{0}=R, & \sigma^{i}=R n^{i} \\
\lambda^{0}=\gamma_{R}, & \lambda^{i}=\gamma v_{R}^{i},
\end{array}
$$

where $R=\left|\mathbf{x}-\mathbf{z}\left(s_{R}\right)\right|=t-z^{0}\left(s_{R}\right)$ is the retarded spatial distance, $\mathbf{v}_{R}$ is the retarded velocity, $\gamma_{R}=\left(1-v_{R}^{2}\right)^{-1 / 2}$, and $\mathbf{n}$ is a unit spatial vector pointing toward the retarded position of the source. In "propagation-delayed Newtonian gravity," aberration appears as the fact that the force is directed along $\mathbf{n}$, and not along the vector pointing toward the "instantaneous" position of the source.

With these conventions, the Liénard-Wiechert potential in Maxwell's electrodynamics can be written as 10

$$
A^{\mu}=\frac{e}{r} \lambda^{\mu}\left(s_{R}\right)
$$

Using standard identities [2,11] obtained from eqn. (1.3), one obtains a field strength tensor

$$
F_{\mu \nu}=\partial_{\mu} A_{\nu}-\partial_{\nu} A_{\mu}=\frac{e}{r^{3}}\left(1-\sigma^{\rho} \frac{d \lambda_{\rho}}{d s}\right)\left(\sigma_{\mu} \lambda_{\nu}-\sigma_{\nu} \lambda_{\mu}\right)+\frac{e}{r^{2}}\left(\sigma_{\mu} \frac{d \lambda_{\nu}}{d s}-\sigma_{\nu} \frac{d \lambda_{\mu}}{d s}\right) .
$$


In particular, eqn. (1.5) implies that the electric field can be written as

$$
E^{i}=F^{i 0}=\frac{e}{\gamma_{R}^{2} R^{2}\left(1-\mathbf{n} \cdot \mathbf{v}_{R}\right)^{3}}\left(n^{i}-v_{R}^{i}\right)+\text { radiative terms }
$$

where the omitted "radiative" terms depend explicitly on acceleration and fall off as $1 / R$ rather than $1 / R^{2}$.

Note that every term in eqns. (1.7) $-(\sqrt{1.8})$ is retarded, and that nothing depends on the "instantaneous" position or direction of the source. The potential (1.6) similarly depends only on purely retarded quantities; while a different gauge choice such as Coulomb gauge may lead to an "instantaneous" term in the potential, this is illusory, since all physical quantities will continue to depend only on retarded characteristics of the source [12].

Nevertheless, the direction of the nonradiative "Coulomb" force in (1.8) is

$$
n^{i}-v_{R}^{i}=\left(1-\mathbf{n} \cdot \mathbf{v}_{R}\right)\left(n^{i}+\left(t-z^{0}\left(s_{R}\right)\right) \frac{d n^{i}}{d t}\right) .
$$

The second term in this expression is essentially a linear extrapolation from the retarded direction $n^{i}$ toward the "instantaneous" direction. In particular, for a charge in uniform motion it is easy to check that $n^{i}-v_{R}^{i}$ points toward the "instantaneous" position, so the effects of aberration are exactly canceled.

Does eqn. (1.8) imply that the electric field propagates instantaneously? Clearly not. In particular, if a uniformly moving charge suddenly stops at position $z\left(s_{0}\right)$, the field at a distant location $x$ will continue to point toward its "extrapolated" position - even though the charge never actually reaches that position - until the time $t-z\left(s_{0}\right)$ that it takes for light to travel from $z\left(s_{0}\right)$ to $x$. At that time, the field will abruptly switch direction to point toward the true position of the source. This sudden change in the field, propagating outward from $z\left(s_{0}\right)$ at the speed of light, is what we mean by the electromagnetic radiation of an accelerated charge. (For a simple derivation of electromagnetic radiation as the retarded effect of the changing Coulomb field of an accelerated charge, see Appendix B of Ref. [13].) One could, of course, try to formulate an alternative model in which the Coulomb field acted instantaneously, but only at the expense of "deunifying" Maxwell's equations and breaking the connection between electric fields and electromagnetic radiation.

\section{Aberration in Gravity}

If we try to extend the arguments of the preceding section to general relativity, we face two subtleties. First, there is no preferred time-slicing in general relativity, and thus no unique definition of an "instantaneous" direction. For weak fields, we can use the nearly flat background to define a nearly Minkowski coordinate system, but we must expect ambiguities of order $v^{2}$. Second, we cannot simply require by fiat that a massive source accelerate. The Einstein field equations are consistent only when all gravitational sources move along the trajectories determined by their equations of motion, and in particular, we can consistently represent an accelerated source only if we include the energy responsible for its acceleration. 
Fortunately, an exact solution for such an accelerated source exists. Kinnersley's "photon rocket" [2,11, 14 represents a mass with an arbitrary acceleration brought about by the nonisotropic emission of electromagnetic radiation. Its metric, in the notation of the preceding section, is

$$
g_{\mu \nu}=\eta_{\mu \nu}-\frac{2 G m\left(s_{R}\right)}{r^{3}} \sigma_{\mu} \sigma_{\nu}
$$

This metric contains four arbitrary functions of time: a time-varying mass $m$ and the three independent components of the acceleration $d \lambda^{\mu} / d s$. In general, it has a nonvanishing stressenergy tensor proportional to $\sigma_{\mu} \sigma_{\nu}$, representing radiation or null dust streaming out from the world line $C$; it reduces to the Schwarzschild metric, with a vanishing stress-energy tensor, when $m$ is constant and $C$ is a straight line.

A test particle in the spacetime (2.1) will travel along a geodesic. If we use the flat metric $m=0$ to define background Minkowski coordinates, the "acceleration" of such a particle, in Newtonian language, is determined by the connection $\Gamma_{\mu \nu}^{\rho}$. In particular, if the particle is initially at rest, its "acceleration" is $-\Gamma_{00}^{i}$. A long but routine computation yields

$$
\begin{aligned}
\Gamma_{\mu \nu}^{\rho}= & -\frac{2 G m}{r^{3}} \eta_{\mu \nu} \sigma^{\rho}+\frac{G m}{r^{4}}\left(3 \lambda_{\mu} \sigma_{\nu} \sigma^{\rho}+3 \lambda_{\nu} \sigma_{\mu} \sigma^{\rho}-\sigma_{\mu} \sigma_{\nu} \lambda^{\rho}\right) \\
& -\frac{3 G m}{r^{5}}\left(1-\sigma_{\tau} \frac{d \lambda^{\tau}}{d s}\right) \sigma_{\mu} \sigma_{\nu} \sigma^{\rho}-\frac{2 G^{2} m^{2}}{r^{6}} \sigma_{\mu} \sigma_{\nu} \sigma^{\rho}-\frac{1}{r^{4}} G \frac{d m}{d s} \sigma_{\mu} \sigma_{\nu} \sigma^{\rho},
\end{aligned}
$$

and in particular,

$$
\begin{aligned}
\Gamma_{00}^{i}=\frac{G m}{R^{2}} \frac{1}{\gamma_{R}^{3}\left(1-\mathbf{n} \cdot \mathbf{v}_{R}\right)^{5}}[ & \left(1-2 \mathbf{n} \cdot \mathbf{v}_{R}-2\left(\mathbf{n} \cdot \mathbf{v}_{R}\right)^{2}+3 v_{R}^{2}\right) n^{i}-\left(1-\mathbf{n} \cdot \mathbf{v}_{R}\right) v_{R}^{i} \\
& \left.-\frac{2 G m}{R} \frac{1}{\gamma_{R}^{3}\left(1-\mathbf{n} \cdot \mathbf{v}_{R}\right)} n^{i}\right]+ \text { radiative terms. }
\end{aligned}
$$

As in the electromagnetic case (1.8), the leading nonradiative term in (2.3) is proportional to $n^{i}-v_{R}^{i}$, so to lowest order there is no aberration. Now, however, there are additional corrections of higher order in $v$. It is not hard to show that the effect of these corrections is to further "extrapolate" from the retarded position toward the "instantaneous" position. Indeed, one finds that

$$
\left.\Gamma_{00}^{i}=\frac{G m}{R^{2}} \frac{1}{\gamma_{R}^{2}\left(1-\mathbf{n} \cdot \mathbf{v}_{R}\right)^{2}}\left[\left(1+\epsilon_{1}\right) \eta^{i}-\frac{2 G m}{R} \frac{1}{\gamma_{R}^{4}\left(1-\mathbf{n} \cdot \mathbf{v}_{R}\right)^{4}} n^{i}+\epsilon_{2} v_{R}^{i}\right)\right]+ \text { radiative terms }
$$

where

$$
\eta^{i}=n^{i}+\left(t-z^{0}\left(s_{R}\right)\right) \frac{d n^{i}}{d t}+\frac{1}{2}\left(t-z^{0}\left(s_{R}\right)\right)^{2} \frac{d^{2} n^{i}}{d t^{2}}
$$

and $\epsilon_{1}$ and $\epsilon_{2}$ are of order $v^{2}$. In other words, the gravitational acceleration is directed toward the retarded position of the source quadratically extrapolated toward its "instantaneous" position, up to small nonlinear terms and corrections of higher order in velocities.

*Note that there is a sign error in Ref. [2]. 
Does eqn. (2.4) imply that gravity propagates instantaneously? As in the case of electromagnetism, it clearly does not. Every term in the connection $\Gamma_{\mu \nu}^{\rho}$ depends only on the retarded position, velocity, and acceleration of the source; despite Van Flandern's claim to the contrary [15], there is no dependence, implicit or explicit, on the "instantaneous" direction to the source. Indeed, the vector (2.5) does not point toward the "instantaneous" position of the source, but only toward its position extrapolated from this retarded data. In particular, as in Maxwell's theory, if a source abruptly stops moving at a point $z\left(s_{0}\right)$, a test particle at position $x$ will continue to accelerate toward the extrapolated position of the source until the time it takes for a signal to propagate from $z\left(s_{0}\right)$ to $x$ at light speed.

A similar result can be obtained in general relativity by evaluating the gravitational field of a boosted black hole [16], or more generally by systematically approximating the solution of the two-body problem [17]. As in the case considered here, the gravitational interaction propagates at the speed of light, but velocity-dependent terms in the interaction nearly cancel the effect of aberration. Indeed, it can be rigorously proven that no gravitational influence in general relativity can travel faster than the speed of light [18].

It is worth noting that the cancellation between aberration and velocity-dependent terms in general relativity is not quite exact. If gravity could be described exactly as an instantaneous, central interaction, the mechanical energy and angular momentum of a system such as a binary pulsar would be exactly conserved, and orbits could not decay. In general relativity, the gravitational radiation reaction appears as a slight mismatch between the effects of aberration and the extra noncentral terms in the equations of motion [17]. One could again try to formulate an alternative theory in which gravity propagated instantaneously, but, as in electromagnetism, only at the expense of "deunifying" the field equations and treating gravity and gravitational radiation as independent phenomena.

\section{Is the Cancellation a Miracle?}

We have seen that the observed lack of aberration in gravitational interactions need not imply an infinite propagation speed, but can be explained as the effect of velocity-dependent terms in the interaction. There is still something to understand, though: a cancellation as exact as that of eqns. (1.9) and (2.5) must surely have a more fundamental origin.

A starting point is Lorentz invariance. As Poincaré first observed, any Lorentz-invariant model of gravitation necessarily requires additional velocity-dependent interactions, which can provide "a more or less perfect compensation" for the effects of aberration [6, 7]. Indeed, Poincaré showed in Ref. [7] that for a Lorentz-invariant model of gravity with light-speed propagation, a correct Newtonian limit, and forces that depend only on positions and velocities, one can choose to eliminate all terms of order $v / c$, so that the deviations from Newtonian gravity are at most of order $v^{2} / c^{2}$. Poincaré did not actually demonstrate that the cancellation of terms of order $v / c$ is necessary. but he showed that aberration terms can be naturally excluded without doing violence to the theory.

\footnotetext{
${ }^{\dagger}$ In the discussion after sect. 9, eqn. (9) of Ref. [], Poincaré's choice of setting the parameter $\beta$ to zero excluded terms of order $v / c$; a reintroduction of $\beta$ would restore such terms.
} 
Consider, for example, the simplest Lorentz-invariant scalar theory of gravity. The naive choice for a retarded Newtonian potential would be $\varphi=m / R$, where $R$ is the propagationdelayed distance (1.5). The gradient $\nabla \varphi$ is proportional to $\mathbf{n}$, and exhibits aberration at order $v / c$. But $\varphi$ is not Lorentz invariant; the simplest invariant version is $\phi=m / r$, where $r$ is the retarded distance (1.4). It is easy to show that

$$
\begin{aligned}
\nabla \phi & =-\frac{\gamma_{R} m}{r^{2}} \frac{1}{\left(1-\mathbf{n} \cdot \mathbf{v}_{R}\right)}\left[\left(1-\mathbf{n} \cdot \mathbf{v}_{R}\right) \mathbf{v}_{R}-\left(1-v_{R}^{2}\right) \mathbf{n}\right]+\text { radiative terms } \\
& =\frac{\gamma_{R} m}{r^{2}}\left(\mathbf{n}+\left(t-z^{0}\left(s_{R}\right)\right) \frac{d \mathbf{n}}{d t}\right)+\frac{\gamma_{R} m}{r^{2}} \frac{1}{\left(1-\mathbf{n} \cdot \mathbf{v}_{R}\right)} \mathbf{v}_{R} \times\left(\mathbf{v}_{R} \times \mathbf{n}\right)+\text { radiative terms. }
\end{aligned}
$$

Thus up to terms of order $v^{2} / c^{2}$, the force points not toward the retarded position of the source, but toward the "linearly extrapolated" retarded direction (1.9); the extra velocity dependence in $r$ eliminates aberration at order $v / c$.

As Van Flandern has stressed, though, astronomical observations require a more complete cancellation: aberration terms of order $v^{3} / c^{3}$ must be eliminated as well. To understand such a cancellation, we can stand the argument of Ref. [1] on its head. As that paper emphasized, a retarded purely central force with no velocity-dependent terms inevitably leads to the drastic nonconservation of orbital ("mechanical") angular momentum and energy in a binary system. But by Noether's theorem, any theory derived from a Lagrangian invariant under rotations and time translations must conserve total angular momentum and energy. For an isolated, bound system, this is only possible if changes in mechanical angular momentum and energy are balanced by changes in the angular momentum and energy of radiation.

For electromagnetism, conservation of charge implies that there can be no monopole radiation, and the power radiated in dipole radiation is proportional to $\left|d^{2} \mathbf{d} / d t^{2}\right|^{2}$, where $\mathbf{d}$ is the electric dipole moment of the source. Since the first derivative $d \mathbf{d} / d t$ is proportional to the velocity, a charge moving at a constant velocity can radiate no angular momentum or energy. Hence at least to first order in velocity, any nonconservation of mechanical angular momentum and energy due to finite propagation speed must be compensated by additional (velocity-dependent) terms in the interaction.

To elaborate this argument, observe that by dimensional analysis, the radiated power is of the form $P \sim\left|d^{2} \mathbf{d} / d t^{2}\right|^{2} c^{-3} \sim e^{2} a^{2} / c^{3}$, where $a$ is the acceleration of the source. Moreover, the virial theorem implies that $m v^{2} \sim e^{2} / r \sim$ mar. Thus

$$
P \sim \frac{e^{2}}{r^{2}} \frac{v^{4}}{c^{3}}=F_{\mathrm{Coulomb}} v \cdot \frac{v^{3}}{c^{3}}
$$

where $F_{\text {Coulomb }}$ is the lowest-order, "instantaneous" Coulomb force. If we want this radiated energy to balance the retardation-induced nonconservation of mechanical energy, the terms responsible for this nonconservation - the velocity-dependent corrections to $F_{\text {Coulomb }}$ - must first appear at order $v^{3} / c^{3}$, and "aberration" effects must cancel at lower orders. The exact mechanism for this cancellation may vary from theory to theory, but its existence is guaranteed by Noether's theorem, and we can be certain that it will appear in any field equations derived from an appropriately invariant action. 
For gravity, conservation of momentum and angular momentum also rule out dipole radiation [19]. The lowest order gravitational radiation is quadrupolar, and the radiated power goes as $\left|d^{3} \mathbf{Q} / d t^{3}\right|^{2}$, where $\mathbf{Q}$ is the mass quadrupole moment of the source. A source with a constant second derivative of $\mathbf{Q}$ can therefore radiate no angular momentum or energy, and any nonconservation of mechanical angular momentum and energy must again be canceled by additional terms in the interaction. The second derivative $d^{2} \mathbf{Q} / d t^{2}$ involves terms proportional to acceleration and to the square of the velocity, so the cancellation must occur at a higher order than it did for electromagnetism. Dimensional analysis now yields

$$
P \sim F_{\mathrm{Newton}} v \cdot \frac{v^{5}}{c^{5}}
$$

so this cancellation must be present up to order $v^{3} / c^{3}$, in agreement with observation.

Note that the dipolar nature of electromagnetic radiation is intimately tied to the fact that the electromagnetic interaction is vectorial (spin 1) [20. Similarly, the quadrupolar nature of gravitational radiation is tied to the traceless tensorial (spin 2) form of the interaction. The case of a scalar interaction is a bit more subtle. For a non-Lorentz invariant theory, conservation laws place no restrictions on radiation, and monopole radiation should dominate, permitting aberration at all orders. For a Lorentz-invariant theory, though, the standard coupling of a scalar field to matter dictates that the monopole moment has the form $m=m_{0} F(\phi)$, so $\dot{m} \sim \dot{\phi}$, which is suppressed by the equations of motion [21. The leading contribution to energy loss thus comes from dipole radiation, and as with electrodynamics, aberration terms first appear at order $v^{3} / c^{3}$. This is the case for the interaction (3.1).

Finally, let us return to the question asked in Ref. [1]: what do experiments say about the speed of gravity? The answer, unfortunately, is that so far they say fairly little. In the absence of direct measurements of propagation speed, observations must be filtered through theory, and different theoretical assumptions lead to different deductions. In particular, while the observed absence of aberration is consistent with instantaneous propagation (with an extra interaction somehow added on to explain the gravitational radiation reaction), it is also consistent with the speed-of-light propagation predicted by general relativity.

Within the framework of general relativity, though, observations do give an answer. The Einstein field equations contain a single parameter $c_{g}$, which describes both the speed of gravitational waves and the "speed of gravity" occurring in the expression for aberration and in the velocity-dependent terms in the interaction. This parameter appears in the gravitational radiation reaction in the form $c_{g}^{-5}$, as in eqn. (3.3), and the success of the theory in explaining the orbital decay of binary pulsars implies that $c_{g}=c$ at the $1 \%$ level or better [22].

\section{Acknowledgements}

This work was supported in part by Department of Energy grant DE-FG03-91ER40674.

\footnotetext{
$\ddagger$ Note that terms of even order in $v / c$ are invariant under time reversal, and do not lead to secular changes, though strictly speaking one must check that there are no time-reversal-invariant effects with very long periods, which can mimic secular changes.
} 


\section{References}

[1] T. Van Flandern, Phys. Lett. A250 (1998) 1.

[2] W. Kinnesley, Phys. Rev. 186 (1969) 1335.

[3] I. J. Good, Am. J. Phys. 43 (1975) 640.

[4] A. P. Lightman, W. H. Press, R. H. Price, and S. A. Teukolsky, Problem Book in Relativity and Gravitation (Princeton University Press, Princeton, 1975).

[5] P. S. Laplace, A Treatise in Celestial Mechanics, Volume IV, Book X, Chapter VII, translated by N. Bowditch (Chelsea, New York, 1966).

[6] H. Poincaré, C. R. Acad. Sci. Paris 140 (1905) 104.

[7] H. Poincaré, Rend. Circ. Matem. Palermo 21 (1906) 129.

[8] For a related argument focusing on the electromagnetic case and its analogy with gravitation, see G. E. March and C. Nissim-Sabat, Phys. Lett. A262 (1999) 27.

[9] R. Feynman, R. B. Leighton, and M. L. Sands, The Feynman Lectures on Physics, Volume II, Chapter 21 (Addison-Wesley, Redwood City, 1989).

[10] J. D. Jackson, Classical Electrodynamics (Wiley, New York, 1975).

[11] T. Damour, Class. Quantum Grav. 12 (1995) 725.

[12] O. L. Brill and B. Goodman, Am. J. Phys. 35 (1967) 832.

[13] E. M. Purcell, Electricity and Magnetism (MacGraw-Hill, New York, 1985).

[14] W. B. Bonnor, Class. Quantum Grav. 11 (1994) 2007.

[15] T. Van Flandern, Phys. Lett. A62 (1999) 261.

[16] M. Ibison, H. E. Puthoff, and S. R. Little, preprint physics/9910050.

[17] T. Damour, in 300 Years of Gravitation, edited by S. W. Hawking and W. Israel (Cambridge University Press, Cambridge, 1987).

[18] R. J. Low, Class. Quantum Grav. 16 (1999) 543.

[19] C. W. Misner, K. S. Thorne, and J. A. Wheeler, Gravitation, Section 36.1 (W. H. Freeman, San Francisco, 1973).

[20] W. E. Couch and E. T. Newman, J. Math. Phys. 13 (1972) 929.

[21] T. Damour and G. Esposito-Farèse, Class. Quantum Grav. 9 (1992) 2093.

[22] J. H. Taylor, Rev. Mod. Phys. 66 (1994) 711. 